\title{
Compressible bubbles in Stokes flow
}

\author{
By DARREN G. CROWDY \\ Department of Mathematics, Imperial College of Science, Technology and Medicine, \\ 180 Queen's Gate, London, SW7 2BZ, UK
}

(Received 29 January 2002 and in revised form 16 September 2002)

The problem of a two-dimensional inviscid compressible bubble evolving in Stokes flow is considered. By generalizing the work of Tanveer \& Vasconcelos (1995) it is shown that for certain classes of initial condition the quasi-steady free boundary problem for the bubble shape evolution is reducible to a finite set of coupled nonlinear ordinary differential equations, the form of which depends on the equation of state governing the relationship between the bubble pressure and its area. Recent numerical calculations by Pozrikidis (2001) using boundary integral methods are retrieved and extended. If the ambient pressures are small enough, it is shown that bubbles can expand significantly. It is also shown that a bubble evolving adiabatically is less likely to expand than an isothermal bubble.

\section{Introduction}

In a recent paper, Pozrikidis (2001) considers the problem of an inviscid twodimensional compressible bubble evolving in an ambient Stokes flow. The motivation is to establish any effects on the size of the bubble induced by the fluid pressures exerted on the bubble due to the ambient flow. Using dynamical simulations based on numerical boundary integral calculations, Pozrikidis (2001) finds that in the case of a bubble placed in pure shear or pure strain, after an initial period of contraction from a circular initial shape, the bubble eventually settles into a steady state which has a greater area than that of the initial circular bubble. These numerical calculations require the computation of certain strongly singular integrals. To test the code, Pozrikidis (2001) makes use of a class of exact solutions due to Tanveer \& Vasconcelos (1995) which are relevant in the special case when the pressure in the bubble is assumed to be identically zero.

In this note, it is shown that the theory of exact solutions expounded by Tanveer \& Vasconcelos (1995) can be generalized to include the case of a compressible bubble evolving according to some equation of state linking the bubble pressure evolution to the bubble area evolution. In this way, for wide classes of initial conditions, the problem can be reduced to the integration of a finite system of coupled ordinary differential equations, the precise form of which depends on the assumed equation of state. It is shown that the results obtained by Pozrikidis (2001) using boundary integral calculations can be retrieved by integrating two coupled nonlinear ordinary differential equations. These two equations must be integrated numerically but the procedure is straightforward. This reformulation not only provides a useful check on the boundary integral calculations (which require the computation of hypersingular integrals Pozrikidis 2001) but enables the problem to be solved much more readily and with much smaller demands on computational capacity. Using the exact solutions, this note $(a)$ includes a wider range of calculations to corroborate the conclusion reached 
by Pozrikidis (2001) that significant area changes in the bubbles are only observed when the ambient pressure is comparable with, or smaller than, the capillary pressure due to surface tension; $(b)$ assesses the effect of the assumed equation of state inside the bubble by comparing results obtained assuming an isothermal bubble (as done by Pozrikidis 2001) with those obtained when the bubble is assumed to change its area adiabatically.

\section{Mathematical formulation}

Consider the quasi-steady evolution of an inviscid bubble surrounded by an infinite expanse of very viscous fluid, with viscosity $\mu$, with surface tension acting on the bubble boundary. Let the velocity field in the fluid have components $\boldsymbol{u}=(u, v)$. Introducing a streamfunction $\psi(x, y)$, then

$$
\boldsymbol{u} \equiv(u, v)=\left(\psi_{y},-\psi_{x}\right),
$$

where subscripts denote partial derivatives. Under the assumption of Stokes flow in the surrounding fluid, it is known that

$$
\nabla^{4} \psi=0,
$$

everywhere in the fluid. Let $p(x, y, t)$ denote the fluid pressure and $p_{B}(t)$ the uniform bubble pressure at time $t$. On the bubble boundary the stress condition is

$$
-p n_{j}+2 \mu e_{j k} n_{k}=\gamma \kappa n_{j}-p_{B}(t) n_{j},
$$

where $\kappa$ is the surface curvature and $\gamma$ is the surface tension; $e_{i j}$ denotes the fluid strain tensor defined by

$$
e_{i j}=\frac{1}{2}\left(\frac{\partial u_{i}}{\partial x_{j}}+\frac{\partial u_{j}}{\partial x_{i}}\right)
$$

and $n_{i}$ is the $i$ th component of the normal $\boldsymbol{n}$ to the boundary. Under the assumption of quasi-steady evolution, the kinematic condition is that

$$
\boldsymbol{u} \cdot \boldsymbol{n}=V_{n},
$$

where $V_{n}$ is the normal velocity of the interface. Equation (2.5) governs the subsequent evolution of the interface. Let the initial bubble area be $\pi a_{0}^{2}$. In what follows, velocities are rescaled by $\gamma / \mu$, pressures by $\gamma / a_{0}$ and length and time with $a_{0}$ and $a_{0} \mu / \gamma$ respectively. To complete the formulation of the problem, conditions in the fluid at large distances from the bubble are required. Following Tanveer \& Vasconcelos (1995), the velocity field at infinity is assumed to have the form

$$
\boldsymbol{u}=\Gamma \cdot \boldsymbol{x}+O(1 /|\boldsymbol{x}|)
$$

as $|\boldsymbol{x}| \rightarrow \infty$ where

$$
\Gamma \equiv \frac{1}{2}\left(\begin{array}{cc}
\alpha_{0} & \beta_{0}-\omega_{0} \\
\beta_{0}+\omega_{0} & -\alpha_{0}
\end{array}\right) .
$$

In (2.7), $\omega_{0}$ denotes the vorticity of the external flow while $\alpha_{0}$ and $\beta_{0}$ characterize its strain rate.

\subsection{Complex variable formulation}

To make progress, the equations above are rewritten in terms of the standard complex variables $z=x+\mathrm{i} y$ and $\bar{z}=x-\mathrm{i} y$. The general solution of (2.2) at each instant can 
be written as

$$
\psi=\operatorname{Im}[\bar{z} f(z, t)+g(z, t)]
$$

where $f(z, t)$ and $g(z, t)$ are functions (to be determined) that are analytic everywhere in the fluid region $D(t)$. In terms of these Goursat functions, the following relations can easily be established (cf. Tanveer \& Vasconcelos 1995):

$$
\begin{gathered}
p-\mathrm{i} \omega=4 f^{\prime}(z, t), \\
u+\mathrm{i} v=-f(z, t)+z \bar{f}^{\prime}(\bar{z}, t)+\bar{g}^{\prime}(\bar{z}, t), \\
e_{11}+\mathrm{i} e_{12}=z \bar{f}^{\prime \prime}(\bar{z}, t)+\bar{g}^{\prime \prime}(\bar{z}, t),
\end{gathered}
$$

where $\omega$ denotes the fluid vorticity. Let $s$ be the arclength traversed in a clockwise direction around the bubble boundary. It can be shown that the stress boundary condition can be written in the form

$$
f(z, t)+z \bar{f}^{\prime}(\bar{z}, t)+\bar{g}^{\prime}(\bar{z}, t)=-\mathrm{i} \frac{z_{s}}{2}+\frac{p_{B}(t) z}{2}
$$

while the complex kinematic condition is

$$
\operatorname{Im}\left[\left(z_{t}+2 f-\frac{p_{B}(t) z}{2}\right) \bar{z}_{s}\right]=-\frac{1}{2} .
$$

A time-dependent conformal mapping $z(\zeta, t)$ from the unit disk in a parametric $\zeta$ plane to the fluid region exterior to the bubble is now introduced. The circle $|\zeta|=1$ maps to the bubble boundary. In terms of this conformal mapping, (2.13) can be rewritten as

$$
\operatorname{Re}\left[\frac{\left(z_{t}+2 f-\frac{1}{2} p_{B}(t) z\right)}{\zeta z_{\zeta}}\right]=\frac{1}{2\left|z_{\zeta}\right|},
$$

where we have used the fact that

$$
z_{s}=\frac{1}{\bar{z}_{s}}=\frac{\mathrm{i} \zeta z_{\zeta}}{\left|z_{\zeta}\right|} \quad \text { on }|\zeta|=1 .
$$

Using the Poisson integral formula for the unit $\zeta$-disk, then for points $\zeta$ inside the unit $\zeta$-disk,

$$
z_{t}(\zeta, t)+2 F(\zeta, t)-\frac{p_{B}(t) z(\zeta, t)}{2}=\zeta[I(\zeta, t)+\mathrm{i} C(t)] z_{\zeta}(\zeta, t),
$$

where $F(\zeta, t) \equiv f(z(\zeta, t), t)$ and

$$
I(\zeta, t)=\frac{1}{4 \pi \mathrm{i}} \oint_{\left|\zeta^{\prime}\right|=1} \frac{\mathrm{d} \zeta^{\prime}}{\zeta^{\prime}}\left[\frac{\zeta^{\prime}+\zeta}{\zeta^{\prime}-\zeta}\right] \frac{1}{\left|z_{\zeta}\left(\zeta^{\prime}, t\right)\right|} .
$$

$C(t)$ is constant in space (but is possibly time-dependent) and is determined by the far-field flow conditions. Equation (2.16) is the generalization of equation (31) of Tanveer \& Vasconcelos (1995). Similarly, defining

$$
G(\zeta, t) \equiv g^{\prime}(z(\zeta, t), t)
$$

the stress condition becomes

$$
G(\zeta, t)=-\bar{F}\left(\zeta^{-1}, t\right)-\bar{z}\left(\zeta^{-1}, t\right) \frac{F_{\zeta}(\zeta, t)}{z_{\zeta}(\zeta, t)}+\frac{\bar{z}_{\zeta}^{1 / 2}\left(\zeta^{-1}, t\right)}{2 \zeta z_{\zeta}^{1 / 2}(\zeta, t)}+\frac{p_{B}(t) \bar{z}\left(\zeta^{-1}, t\right)}{2} .
$$


Given that the far-field velocity has the form (2.6), the asymptotic conditions on $f(z)$ and $g(z)$ as $z \rightarrow \infty$ are

$$
\begin{aligned}
& f(z) \sim \frac{1}{4}\left(p_{\infty}-\mathrm{i} \omega_{0}\right) z+O(1), \\
& g^{\prime}(z) \sim \frac{1}{2}\left(\alpha_{0}-\mathrm{i} \beta_{0}\right) z+O(1),
\end{aligned}
$$

where $p_{\infty}$ is the fluid pressure at infinity. Equation (2.19) is the generalization of equation (36) of Tanveer \& Vasconcelos (1995) to the case where the bubble pressure is not identically zero but takes some spatially uniform time-evolving value $p_{B}(t)$. It is important to note that equations (31) and (36) of Tanveer \& Vasconcelos (1995) are the two equations which are manipulated to derive classes of exact solutions. Tanveer \& Vasconcelos (1995) devote $\S 3.1$ of their paper to explaining the general mathematical reasons underlying the existence of this class of solutions.

For purposes of generalization to the case where the bubble pressure may vary in time according to some externally specified prescription (i.e. equation of state), the following observation is crucial. Define a modified Goursat function $\hat{f}(z, t)$ via the time-dependent transformation

$$
\hat{f}(z, t) \equiv f(z, t)-\frac{p_{B}(t) z}{4} .
$$

$\hat{f}(z, t)$ shares the same analyticity properties as $f(z, t)$ everywhere inside the fluid. Crucially, its large- $z$ behaviour is also of the same general kind as $f(z, t)$, that is,

$$
\hat{f}(z, t) \sim \frac{1}{4}\left(p_{\infty}-\mathrm{i} \omega_{0}\right) z-\frac{1}{4} p_{B}(t) z \quad \text { as } z \rightarrow \infty .
$$

Now define

$$
\mathscr{F}(\zeta, t) \equiv \hat{f}(z(\zeta, t), t)
$$

Then, under the transformation (2.22), equations (2.16) and (2.19) become

$$
z_{t}(\zeta, t)+2 \mathscr{F}(\zeta, t)=\zeta[I(\zeta, t)+\mathrm{i} C(t)] z_{\zeta}(\zeta, t),
$$

and

$$
G(\zeta, t)=-\overline{\mathscr{F}}\left(\zeta^{-1}, t\right)-\bar{z}\left(\zeta^{-1}, t\right) \frac{\mathscr{F}_{\zeta}(\zeta, t)}{z_{\zeta}(\zeta, t)}+\frac{\bar{z}_{\zeta}^{1 / 2}\left(\zeta^{-1}, t\right)}{2 \zeta z_{\zeta}^{1 / 2}(\zeta, t)} .
$$

Equations (2.25) and (2.26) are now isomorphic to (31) and (36) of Tanveer \& Vasconcelos (1995). Moreover, inspection of $\S 3.1$ of Tanveer \& Vasconcelos (1995) in which their equations (31) and (36) are analysed in detail shows that the existence of the exact solution class depends only on the a priori known analytical structure of (their) $F(\zeta, t)$ and $G(\zeta, t)$ inside the unit $\zeta$-circle. The modified functions $\mathscr{F}(\zeta, t)$ and $G(\zeta, t)$ defined here share exactly the same analyticity structure in the unit $\zeta$-circle as the functions $F(\zeta, t)$ and $G(\zeta, t)$ defined in Tanveer \& Vasconcelos (1995) (including, crucially, those at $\zeta=0$ corresponding to physical infinity). It can thus be concluded that exact solutions to the compressible bubble problem also exist and can be derived using the methods expounded in Tanveer \& Vasconcelos (1995) while taking care to make the transformation embodied in (2.22). This transformation affects the far-field asymptotic flow conditions (and thus the relevant equations governing the motion) but the analytic structure of the conformal mapping solutions established in Tanveer \& Vasconcelos (1995) persists. The exact solutions are given by rational-function conformal mappings depending on a finite set of time-evolving parameters.

To derive the evolution equations for a given rational-function conformal mapping, the procedure is to substitute for $\mathscr{F}(\zeta, t)$ from (2.25) into (2.26). The right-hand side of 
the resulting equation then depends only on the conformal mapping function (and its conjugate function) while the left-hand side is simply $G(\zeta, t)$. The evolution equations for the conformal mapping parameters are obtained by ensuring that $G(\zeta, t)$ has the correct analyticity structure inside the circle $|\zeta| \leqslant 1$. This procedure will be illustrated in the context of the examples of $\S 3$.

\section{Compressible bubbles in a linear flow}

Pozrikidis (2001) considers the example of compressible bubbles in pure shear flow and pure straining flows. For purposes of comparison with the boundary integral calculations of Pozrikidis (2001) the same class of flows, and initial conditions will be examined. In the following calculations, the initial bubble is taken to be purely circular of unit radius. The initial bubble pressure is given by the Young-Laplace formula for a circular bubble in equilibrium under the effects of surface tension with no external flow, i.e.

$$
p_{B_{0}}=p_{\infty}+1 \text {. }
$$

Two different equations of state will be considered. Under the assumption that the bubble is an ideal gas, $p_{B}(t)$ is related to bubble area $A_{B}(t)$ via the formula

$$
p_{B}(t) A_{B}(t)=p_{B_{0}} \pi=\pi\left(p_{\infty}+1\right) .
$$

If the bubble is assumed to change its area adiabatically so that $p / \rho^{\gamma}=$ constant, the equation of state is

$$
p_{B}(t)\left[A_{B}(t)\right]^{\gamma}=p_{B_{0}} \pi^{\gamma}=\pi^{\gamma}\left(p_{\infty}+1\right),
$$

where, for definiteness, we here take $\gamma=1.4$.

The resulting systems of ordinary differential equations are solved using a simple forward Euler method with time step $10^{-3}$. Any integral quantities appearing in the equations are evaluated using a trapezoidal rule taking 512 sample points on the unit $\zeta$-circle. Since the trapezoidal rule gives super-algebraic convergence for periodic functions on smooth domains, we expect the dominant numerical error to be the $O\left(10^{-3}\right)$ global error associated with the forward Euler scheme. This method gives adequate accuracy for present purposes. The results are checked by recalculating with time step equal to $10^{-4}$ and 1024 points in the trapezoidal rule.

\subsection{Compressible bubble in pure shear flow}

Let the far-field flow be

$$
\boldsymbol{u}=(k y, 0)
$$

where the shear rate $k$ is some positive constant. The compressible bubble will evolve, and adjust its area, both as a result of the imposed ambient flow field and the pressure at infinity. The far-field form of $\hat{f}(z)$ and $g^{\prime}(z)$ are given by

$$
\begin{gathered}
\hat{f}(z) \sim \frac{1}{4}\left(p_{\infty}-p_{B}(t)+\mathrm{i} k\right) z+O(1) \quad \text { as } z \rightarrow \infty, \\
g^{\prime}(z) \sim-\frac{1}{2} \mathrm{i} k z+O(1) \quad \text { as } z \rightarrow \infty .
\end{gathered}
$$

The initial conformal map $z_{0}(\zeta)$ from the unit $\zeta$-circle to the initial bubble is

$$
z_{0}(\zeta)=\frac{1}{\zeta}
$$




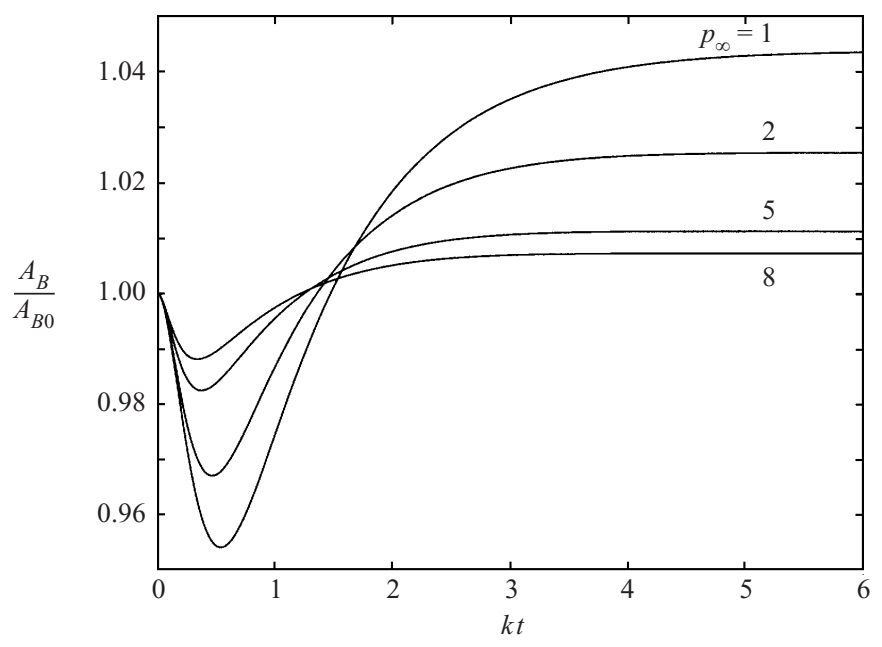

FIGURE 1. Evolution of the reduced area of a compressible bubble in simple shear flow; $k=0.5$ and $p_{\infty}=1,2,5$ and 8.

By the results of $\S 2$, under evolution the time-dependent conformal map has the form

$$
z(\zeta, a(t), b(t))=\frac{a(t)}{\zeta}+b(t) \zeta,
$$

where $a(t)$ can be assumed real (by a rotational degree of freedom in the Riemann mapping theorem) and $b(t)$ is complex in general. By comparison of (3.7) and (3.8) the initial conditions are $a(0)=1$ and $b(0)=0$. For convenience, the dependence of $a(t)$ and $b(t)$ on time will be suppressed. The ordinary differential equations for $a$ and $b$ are found to be

$$
\begin{gathered}
\dot{a}=-a I(0, a, b)-\left(\frac{p_{\infty}-p_{B}(a, b)}{2}\right) a, \\
\dot{b}=-b I(0, a, b)+\left(\frac{p_{\infty}-p_{B}(a, b)}{2}\right) b+\mathrm{i} k(a-b),
\end{gathered}
$$

where

$$
I(0, a, b)=\frac{1}{4 \pi \mathrm{i}} \oint_{|\zeta|=1} \frac{\mathrm{d} \zeta}{\zeta} \frac{1}{\left|z_{\zeta}(\zeta, a, b)\right|},
$$

and the bubble pressure, now denoted $p_{B}(a, b)$ (instead of just $\left.p_{B}(t)\right)$, is

$$
p_{B}(a, b)=\frac{\left(p_{\infty}+1\right)}{\left(a^{2}-|b|^{2}\right)}
$$

in the case of an ideal gas, and

$$
p_{B}(a, b)=\frac{\left(p_{\infty}+1\right)}{\left(a^{2}-|b|^{2}\right)^{\gamma}}
$$

when the bubble evolution is assumed to be adiabatic. Equations (3.12) and (3.13) follow from (3.2) and (3.3) respectively, together with the fact that, for bubbles described by the conformal map (3.8), $A_{B}(t)=\pi\left(a^{2}-|b|^{2}\right)$. Details of the derivation of (3.9)-(3.10) using the fundamental equations (2.25) and (2.26) are given in the Appendix. 


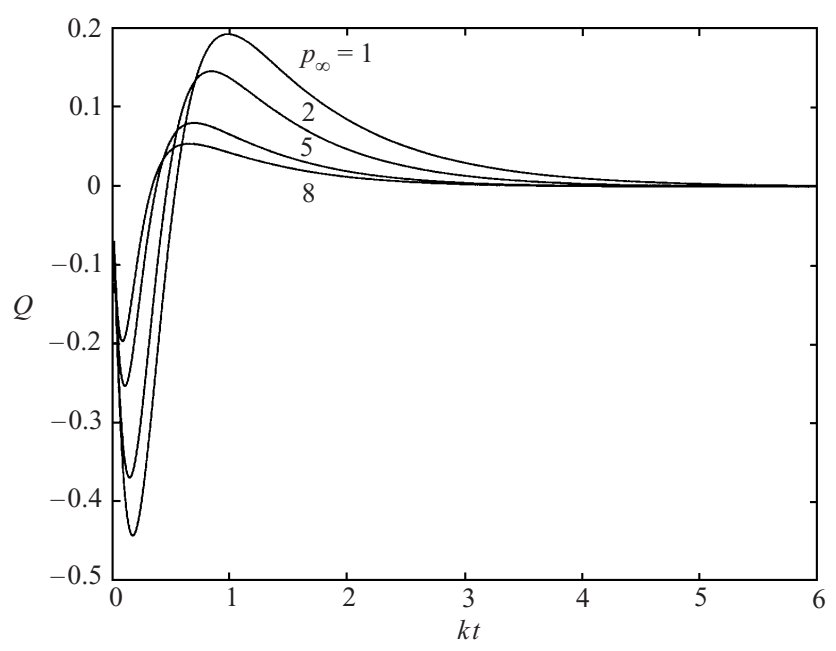

FIGURE 2. Reduced rate of expansion $Q$ of a compressible bubble in simple shear flow; $k=0.5$ and $p_{\infty}=1,2,5$ and 8.

Pozrikidis (2001) defines the initial capillary number $C a_{0}$ as

$$
C a_{0}=\frac{\mu k a_{0}}{\gamma}
$$

where $a_{0}$ is the initial bubble radius. In the present notation, and with the initial bubble taken to be circular with unit radius, the parameter $k$ corresponds exactly to the $C a_{0}$ parameter of Pozrikidis (2001). For purposes of comparison with the results of the boundary integral calculations, in figures 1 and 2 the graphs plotted in figures $7(a)$ and $7(b)$ of Pozrikidis (2001) are recalculated by integration of the equations (3.10) for $a$ and $b$. These graphs show the bubble area $A_{B}(t)$ (rescaled by the initial bubble area $A_{B 0}$ ) and the reduced rate of expansion $Q$ plotted against reduced time $k t . Q$ is determined using the formula

$$
Q=\frac{\dot{A}_{B}(t)}{k a_{0}^{2}}=\frac{\pi}{k a_{0}^{2}}(2 a \dot{a}-b \dot{\bar{b}}-\bar{b} \dot{b}),
$$

combined with the equations in (3.10). Here the bubble is assumed to be isothermal, the capillary number is such that $k=0.5$ and four values of $p_{\infty}=1,2,5$ and 8 are considered. The graphs show good agreement with the boundary integral calculations. For example, the value obtained for the net percentage increase in bubble area at steady state as calculated by the method above (with $p_{\infty}=1$ and $k=0.5$ ) is $4.4 \%$ while that reported by Pozrikidis (2001) using the boundary integral method is just under $4 \%$. The net percentage increase in bubble area at steady state is greatest for the case $p_{\infty}=1$ and appears to decrease as the ambient pressure increases. In order to examine how this overall expansion amount varies as a function of the ambient pressure $p_{\infty}$, figure 3 shows a graph of reduced bubble area at steady state, for fixed capillary number $k=0.5$, as a function of $p_{\infty}$ for a range of positive $p_{\infty}$-values. The relative simplicity of the numerical calculations (compared with the boundary integral methods) facilitates rapid calculation of the overall expansion rate for a wide range of $p_{\infty}$ values. As $p_{\infty}$ draws closer to zero, the net expansion at steady state can reach up to $12.5 \%$. This is much more significant than the value of about $4 \%$ in the case $p_{\infty}=1$. Also shown in figure 3 are the same results in the case where the bubble is taken to change its area adiabatically. In this case, the overall expansion effect is 


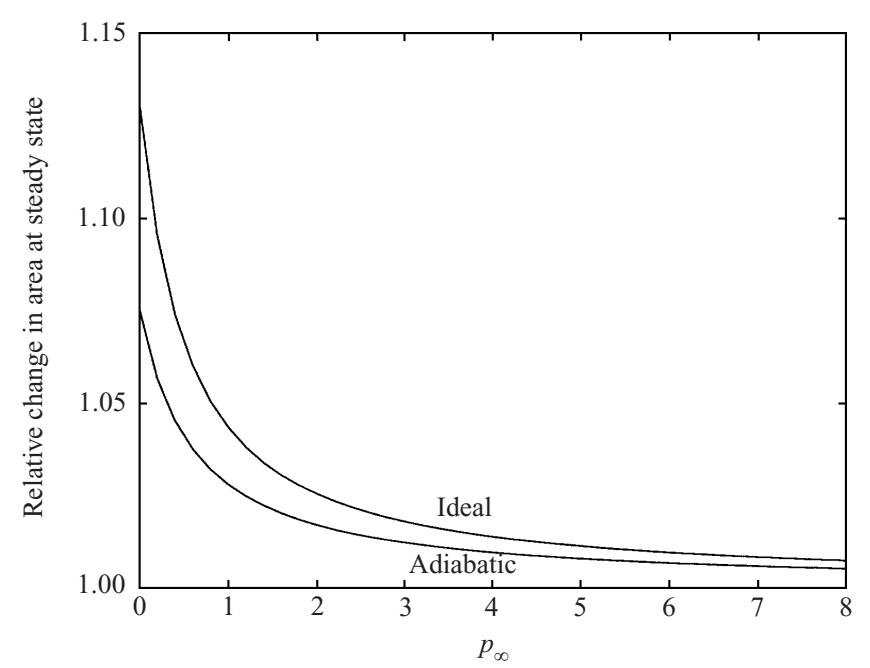

FIGURE 3. Relative change in area at steady state for a compressible bubble in pure shear with $k=0.5$ for different values of the ambient pressure $p_{\infty}$. Results are shown for the two different equations of state.

significantly reduced (by comparison with the isothermal case) at all values of the ambient pressure $p_{\infty}$. In particular, the maximum percentage change observed for positive ambient pressures in this case is about $7.5 \%$ (when $p_{\infty}=0$ ). The equation of state of the compressible bubble can therefore make a significant difference in any expansion effects induced by the ambient flow.

The exact solution structure persists regardless of the choice of the equation of state used to determine $p_{B}(t)$ at each instant. Exact solutions also exist if the shear rate $k$ is taken to be a time-dependent function, i.e. $k=k(t)$. It is also emphasized that large classes of different initial conditions where the initial fluid region maps from the unit $\zeta$-disk via a rational-function conformal map can also be studied using exact solutions. The conformal map remains a rational function under evolution, its poles and zeros evolving in time according to a system of first-order ordinary differential equations.

\subsection{Compressible bubble in pure strain flow}

The case of a bubble in a pure strain flow is also considered by Pozrikidis (2001). Let the far-field flow now be given by

$$
\boldsymbol{u}=(k x,-k y),
$$

where the strain rate $k$ is again taken to be a positive constant. In this case, we expect the bubble to retain a reflectional symmetry about both the $x$ - and $y$-axes which implies that $b(t)$ will be real. The initial conformal map is again given by (3.7) while, for times $t>0$, the results of $\S 2$ imply that the conformal map will retain the form (3.8) under evolution. The ordinary differential equations for $a$ and $b$ are now

$$
\begin{gathered}
\dot{a}=-a I(0, a, b)-\left(\frac{p_{\infty}-p_{B}(a, b)}{2}\right) a, \\
\dot{b}=-b I(0, a, b)+\left(\frac{p_{\infty}-p_{B}(a, b)}{2}\right) b+2 k a,
\end{gathered}
$$

with $I(0, a, b)$ and $p_{B}(a, b)$ given by (3.11) and (3.12) respectively. These equations are obtained in a similar way to the derivation of (3.9)-(3.10). 


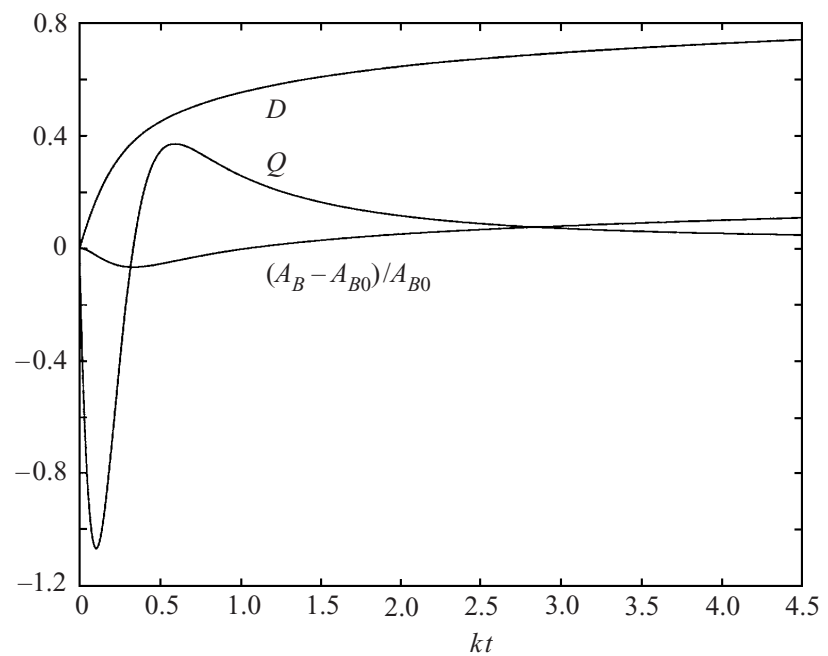

Figure 4. Evolution of a compressible bubble in pure strain with $k=0.3$ and $p_{\infty}=1$.

With $k=0.3$ and $p_{\infty}=1$ (corresponding to the calculations performed by Pozrikidis 2001) the bubble is found to have expanded by over $8 \%$ by the end of the simulation at $k t=3$. This is in contrast with the value of just under $5 \%$ reported by Pozrikidis (2001). Figure 4 shows the evolution of $D, Q$ and $\left(A_{B}-A_{B 0}\right) / A_{B 0}$ with time; the same graph is plotted by Pozrikidis (2001) with $D$ denoting the Taylor deformation parameter, $Q$ the mass flux while $A_{B 0}$ and $A_{B}$ denote the initial bubble area and the bubble area at time $t$. The graph of $Q$ given in figure 10 of Pozrikidis (2001) shows a much less smooth evolution than that shown in the present figure 4 . This is presumably due to numerical errors in the boundary integral simulation. The $3 \%$ difference in the overall change in bubble areas as calculated by the two separate methods is presumably a result of the accumulation of these numerical errors in the boundary integral calculation. As remarked in Pozrikidis (2001), in this case the expansion of the bubble has raised the capillary number so that it is barely outside the regime where a steady-state shape exists. This is evinced by the graph of $Q$ in figure 4 which tends to zero at large times but never quite reaches it.

It is again of interest to examine how the ambient pressure affects the overall expansion at steady state in the case of a bubble in a strain flow. However, as just seen, the existence of a steady state in straining flow is no longer guaranteed but depends on the values of $k$ and $p_{\infty}$. The choice $k=0.25$ is made and it is found, by direct integration of the ordinary differential equations (3.17)-(3.18), that steady states exist at least for $1 \leqslant p_{\infty} \leqslant 8$ in both the ideal and adiabatic cases. Figure 5 therefore shows the reduced area at steady state for this particular range of ambient pressures. Results are plotted for both equations of state. Again, maximum expansion occurs for the lowest value of the ambient pressure considered (that is, $p_{\infty}=1$ ) where an isothermal bubble is found to have expanded by about $6.7 \%$, while a bubble evolving adiabatically expands by just $4.1 \%$. As in the case of a pure ambient shear, isothermal bubbles in a strain flow are found to be more susceptible to expansion at all values of the ambient pressure than bubbles whose area changes adiabatically.

\section{Final remarks}

It has been seen that, when an ambient flow is initiated, a circular bubble disturbed from a Laplace-Young equilibrium expands in the process of adjusting to a modified 


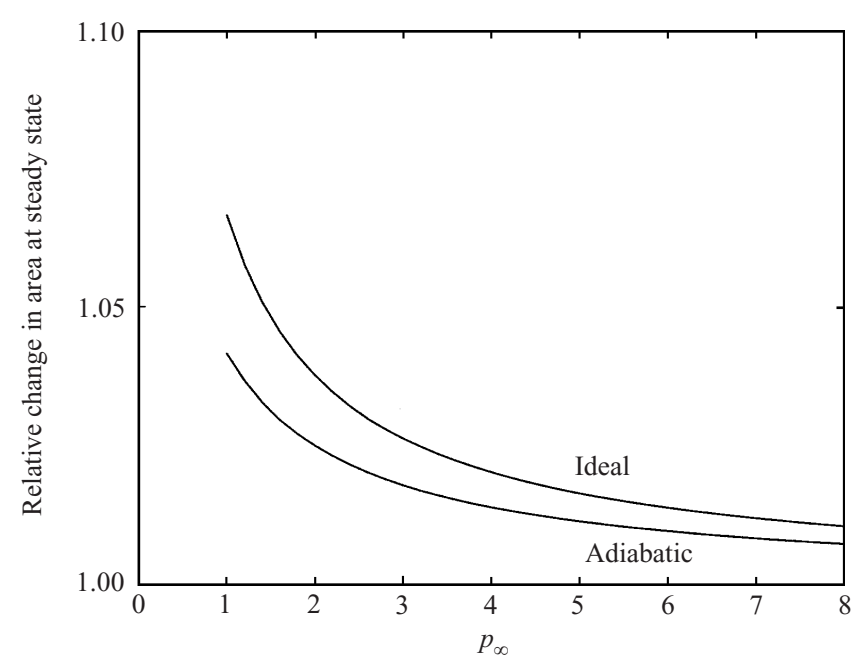

FiguRE 5. Relative change in area at steady state for a compressible bubble in pure strain with $k=0.25$ for a range of ambient pressures $p_{\infty}$ where steady solutions are found to exist. Results are shown for the two different equations of state.

elliptical equilibrium. This adjustment has been shown here to take place via a sequence of intermediate purely elliptical shapes. The results herein imply the existence of many other non-trivial exact solutions to this free boundary problem in addition to those within the class of time-evolving ellipses.

It is worth mentioning a potential application of the present results. The problem of the evolution of inviscid compressible enclosures (i.e. pores or bubbles) in slow viscous flows has previously been considered by Mackenzie \& Shuttleworth (1952). Their study was motivated by the industrially important manufacturing process known as viscous sintering (cf. Kuiken 1990). In the sintering process, a compact of touching particles is heated to a sufficiently high temperature that the particles become mobile. It is then appropriate to model them as regions of very viscous fluid. Once mobile, the driving force of the motion is surface tension which generally leads to densification of the compact as the pores or bubbles close up. Thus, the application naturally leads to a situation where it is of interest to examine the effects of an ambient Stokes flow (i.e. the motion of the surrounding compact) on the size of an enclosed compressible bubble/pore. This is the problem considered here, albeit in the planar case. The observed shrinking of the pores is not in conflict with the present results where the ambient flow is seen to induce an overall bubble/pore expansion. This is because the relevant initial conditions in each case are different. In the sintering application, it is not appropriate to assume an initial Laplace-Young equilibrium as in (3.1) but rather to assume that $p_{B 0}=p_{\infty}$ at the initial instant. Hopper (1990) has considered the shrinkage rate of an isolated constant-pressure bubble, subject to this initial condition, in the case where the driving mechanism is surface tension alone and there is no imposed ambient flow.

Although a fully three-dimensional situation is considered, the theoretical approach of Mackenzie \& Shuttleworth (1952) is phenomenological. Two different physical assumptions regarding the pores are considered in detail: the case of pores at constant uniform pressure and that of pores containing a fixed mass of ideal gas at constant temperature (i.e. pores containing an isothermal gas). To model the effects on a given pore of all surrounding pores in the medium, Mackenzie \& Shuttleworth (1952) 
employ the phenomenological device of an 'equivalent' spherical shell surrounding the pore. While only the case of a single bubble has been considered here, it would interesting to examine whether the exact solution methods and/or the boundary integral methods of Pozrikidis (2001) are extendable to the case of multiple compressible bubbles. This would help to understand the interaction of compressible bubbles with a general ambient Stokes flow without the need for phenomenological models of this kind.

\section{Appendix. Derivation of evolution equations (3.9)-(3.10)}

With the conformal mapping of the form (3.8), as $\zeta \rightarrow 0$, we have

$$
\begin{gathered}
z_{t}(\zeta, t) \sim \frac{\dot{a}}{\zeta}+O(1), \\
\zeta z_{\zeta}(\zeta, t) \sim-\frac{a}{\zeta}+O(1), \\
2 \mathscr{F}(\zeta, t) \sim\left(\frac{\left(p_{\infty}-p_{B}(a, b)+\mathrm{i} k\right)}{2}\right) \frac{a}{\zeta}+O(1) .
\end{gathered}
$$

Substituting these into equation (2.25) and equating coefficients of $\zeta^{-1}$ yields

$$
\dot{a}+\left(\frac{p_{\infty}-p_{B}(a, b)+\mathrm{i} k}{2}\right) a=-a(I(0, a, b)+\mathrm{i} C) .
$$

The real part of (A 4) yields

$$
\dot{a}=-a I(0, a, b)-\left(\frac{p_{\infty}-p_{B}(a, b)}{2}\right) a,
$$

which is precisely the first equation in (3.10), while the imaginary part implies that $C=-k / 2$.

Now, (2.25) implies that

$$
\mathscr{F}(\zeta, t)=\frac{1}{2}\left(-\frac{\dot{a}}{\zeta}-\dot{b} \zeta+\left(b \zeta-\frac{a}{\zeta}\right)[I(\zeta, a, b)-\mathrm{i} k / 2]\right),
$$

and

$$
\overline{\mathscr{F}}\left(\zeta^{-1}, t\right)=\frac{1}{2}\left(-\dot{a} \zeta-\frac{\dot{\bar{b}}}{\zeta}+\left(\frac{\bar{b}}{\zeta}-a \zeta\right)[-I(\zeta, a, b)+\mathrm{i} k / 2]\right),
$$

where we have used the fact that $\bar{I}\left(\zeta^{-1}, a, b\right)=-I(\zeta, a, b)$ for points inside $|\zeta|<1$. As $\zeta \rightarrow 0$

$$
\begin{gathered}
\mathscr{F}(\zeta, t) \sim \frac{1}{2}(-\dot{a}-a[I(0, a, b)-\mathrm{i} k / 2]) \frac{1}{\zeta}+O(1), \\
\overline{\mathscr{F}}\left(\zeta^{-1}, t\right) \sim \frac{1}{2}(-\dot{\bar{b}}-\bar{b}[I(0, a, b)-\mathrm{i} k / 2]) \frac{1}{\zeta}+O(1), \\
G(\zeta, t) \sim-\frac{\mathrm{i} k a}{2 \zeta}+O(1) .
\end{gathered}
$$

But (2.26) multiplied by $z_{\zeta}(\zeta, t)$ has the form

$$
G(\zeta, t) z_{\zeta}(\zeta, t)=-\overline{\mathscr{F}}\left(\zeta^{-1}, t\right) z_{\zeta}(\zeta, t)-\bar{z}\left(\zeta^{-1}, t\right) \mathscr{F}_{\zeta}(\zeta, t)+\frac{\left[z_{\zeta}(\zeta, t) \bar{z}_{\zeta}\left(\zeta^{-1}, t\right)\right]^{1 / 2}}{2 \zeta} .
$$


Substituting (A 10) into (A 11) and equating powers of $\zeta^{-3}$ yields

$$
\mathrm{i} k a^{2}=-a \dot{\bar{b}}-\dot{a} \bar{b}-2 a \bar{b}[I(0, a, b)-\mathrm{i} k / 2],
$$

which, on rearrangement and use of (A 5), yields the second equation in (3.10).

\section{REFERENCES}

Hopper, R. W. 1990 Plane Stokes flow driven by capillarity on a free surface. J. Fluid Mech. 213, $349-375$.

KuIKEN, H. K. 1990 Viscous sintering: the surface-tension-driven flow of a liquid form under the influence of curvature gradients at its surface. J. Fluid Mech. 214, 503-515.

Mackenzie, J. K. \& Shuttleworth, R. 1952 A phenomenological theory of sintering. Proc. Phys. Soc. B 62, 833-852.

PozRIKIDIs, C. 2001 Expansion of a compressible gas bubble in Stokes flow. J. Fluid Mech. 442, $171-189$.

Tanveer, S. \& Vasconcelos, G. 1995 Time-evolving bubbles in two-dimensional Stokes flow. J. Fluid Mech. 301, 325-344. 